PROCEEDINGS OF THE

AMERICAN MATHEMATICAL SOCIETY

Volume 126, Number 1, January 1998, Pages 173-179

S 0002-9939(98)03949-5

\title{
WEAK CONVERGENCE OF INNER SUPERPOSITION OPERATORS
}

\author{
MIKHAIL E. DRAKHLIN AND EUGENE STEPANOV
}

(Communicated by Palle E. T. Jorgensen)

\begin{abstract}
The equivalence of the weak (pointwise) and strong convergence of a sequence of inner superposition operators is proved as well as the criteria for such convergence are provided. Besides, the problems of continuous weak convergence of such operators and of representation of a limit operator are studied.
\end{abstract}

\section{INTRODUCTION}

The study of well-posedness of boundary value problems for functional differential equations most often involves the study of various types of convergence of inner superposition operators [1]. Of particular interest are the pointwise and continuous convergence.

Recall the precise definitions. Let $A_{\nu}: X \rightarrow Y, A: X \rightarrow Y$, where $\nu \in \mathbb{N}$ are the mappings between two Banach spaces $X$ and $Y$. One says that the sequence $A_{\nu}$ converges to $A$

(S) strongly (pointwise), if $A_{\nu} x \rightarrow A x$ in $Y$ for all $x \in X$;

(C) continuously, if $A_{\nu} x_{\nu} \rightarrow A x$ in $Y$ for any norm converging sequence $x_{\nu} \rightarrow x$ in $X$;

(W) weakly (pointwise), if $A_{\nu} x \rightarrow A x$ in $Y$ for all $x \in X$;

(CW) continuously weakly, if $A_{\nu} x_{\nu} \rightarrow A x$ in $Y$ for any weakly converging sequence $x_{\nu} \rightarrow x$ in $X$.

It is clear that in particular for the linear operators $A_{\nu}$

$$
(\mathrm{C}) \Leftrightarrow(\mathrm{S}) \Rightarrow(\mathrm{W}) \Leftarrow(\mathrm{CW}) \text {, }
$$

while in each of the four cases this sequence is necessarily uniformly bounded, the limit operator $A$ being linear and bounded. Note that in general $(\mathrm{W}) \nRightarrow(\mathrm{S})$, which can be observed from considering the example of a sequence of linear operators

$$
A_{\nu}: L^{2}(0,1) \rightarrow L^{2}(0,1), \quad\left(A_{\nu} x\right)(t)=x(t) \sin \nu \pi t .
$$

It converges weakly (pointwise) to a zero operator, but does not converge strongly. Here and in the sequel $L^{p}(0,1)$ (for short, $\left.L^{p}\right)$ stands for the standard Lebesgue space of functions integrable on the interval $(0,1)$ with the power $1 \leq p<\infty$, equipped by the norm $\|x\|_{p}:=\left(\int_{0}^{1}|x(\tau)|^{p} d \tau\right)^{1 / p}$. The same example also asserts

Received by the editors October 23, 1995 and, in revised form, July 3, 1996.

1991 Mathematics Subject Classification. Primary 47B38, 47A67, 34K05.

(C)1998 American Mathematical Society 
that $(\mathrm{W}) \nRightarrow(\mathrm{CW})$ : it is enough to set $x_{\nu}(t):=\sin \nu \pi t$ observing that $A_{\nu} x_{\nu} \dashv 0$ in $L^{2}$.

The continuous weak convergence $(\mathrm{CW})$ deserves a special discussion because of its importance in the study of variational convergence for optimal control problems [2]. Let $X^{\prime}$ and $Y^{\prime}$ stand for the duals of $X$ and $Y$ respectively and denote by $A_{\nu}^{\prime}, A^{\prime}: Y^{\prime} \rightarrow X^{\prime}$ the adjoints of the operators $A_{\nu}, A: X \rightarrow Y$. By $\left(\mathrm{S}^{\prime}\right)$ and $\left(\mathrm{W}^{\prime}\right)$ denote respectively the strong and weak (pointwise) convergences of the sequence $A_{\nu}^{\prime}$ to $A^{\prime}$. One has then the obvious implications

$$
(\mathrm{S}) \Rightarrow(\mathrm{W}) \Leftrightarrow\left(\mathrm{W}^{\prime}\right) \Leftarrow(\mathrm{CW}) \Leftarrow\left(\mathrm{S}^{\prime}\right) .
$$

If, in addition, $X$ is reflexive and $Y$ is a uniformly convex (in particular, Hilbert) space, then

$$
(\mathrm{CW}) \Leftrightarrow\left(\mathrm{S}^{\prime}\right) .
$$

To show this, assume $(\mathrm{CW})$ and consider an arbitrary subsequence of a sequence of operators $\left\{A_{\nu}\right\}$ (for brevity we will use the same index $\nu$ ). As $A_{\nu}^{\prime} y^{\prime} \rightarrow A^{\prime} y^{\prime}$ for each $y^{\prime} \in Y^{\prime}$, then $\left\|A^{\prime} y^{\prime}\right\|_{X^{\prime}} \leq \liminf _{\nu}\left\|A_{\nu}^{\prime} y^{\prime}\right\|_{X^{\prime}}$. On the other hand, according to the Hahn-Banach theorem there is a sequence $\left\{x_{\nu}\right\} \in X$, such that $\left\|x_{\nu}\right\|_{X}=1$ and $\left\langle x_{\nu}, A_{\nu}^{\prime} y^{\prime}\right\rangle=\left\|A_{\nu}^{\prime} y^{\prime}\right\|_{X^{\prime}}$. Extracting a weakly converging subsequence $x_{\nu_{\mu}} \rightarrow x$, $\|x\|_{X} \leq 1$, and recalling that the continuous convergence of a sequence of operators implies the continuous convergence of any of its subsequences to the same limit ([3], Chapter II, $\S 20$, Section VII), one sees that $\left\|A_{\nu_{\mu}}^{\prime} y^{\prime}\right\|_{X^{\prime}} \rightarrow\left\langle x, A^{\prime} y^{\prime}\right\rangle \leq\left\|A^{\prime} y^{\prime}\right\|_{X^{\prime}}$ and hence $A_{\nu_{\mu}}^{\prime} y^{\prime} \rightarrow A^{\prime} y^{\prime}$. Therefore, the whole weakly convergent sequence $\left\{A_{\nu}^{\prime} y^{\prime}\right\} \subset X^{\prime}$ is compact and thus $A_{\nu}^{\prime} y^{\prime} \rightarrow A^{\prime} y^{\prime}$.

In this paper given the sequence of measurable functions $g^{\nu}:[0,1] \rightarrow \mathbb{R}$ and $g$ : $[0,1] \rightarrow \mathbb{R}$, we are interested in the relationship between the convergence properties $(\mathrm{C}),(\mathrm{W})$ and $(\mathrm{S})$ of a sequence of respective linear inner superposition operators $S_{g^{\nu}}: L^{p} \rightarrow L^{q}$ given by the formulae

$$
\left(S_{g^{\nu}} x\right)(t)=\left\{\begin{array}{cl}
x\left(g^{\nu}(t)\right), & g^{\nu}(t) \in[0,1], \\
0, & g^{\nu}(t) \notin[0,1]
\end{array}\right.
$$

to another inner superposition operator $S_{g}: L^{p} \rightarrow L^{q}$ given by

$$
\left(S_{g} x\right)(t)=\left\{\begin{array}{cl}
x(g(t)), & g(t) \in[0,1] \\
0, & g(t) \notin[0,1] .
\end{array}\right.
$$

For the above operators to be well-defined on the classes of measurable functions we are to impose the following conditions on the functions $g^{\nu}$ and $g$ (denoting from now on for the sake of brevity $g^{0}=g$ ):

$$
e \subset[0,1], \text { meas } e=0 \Rightarrow \text { meas }\left(g^{\nu}\right)^{-1}(e)=0, \nu \in \mathbb{N} \cup\{0\} .
$$

In fact, we will show that for the inner superposition operators

$$
(\mathrm{C}) \Leftrightarrow(\mathrm{S}) \Leftrightarrow(\mathrm{W})
$$

and write out the necessary and sufficient conditions for such convergence in terms of the functions $g^{\nu}$. It will be also shown, however, that in general even for such particular operators $(\mathrm{S}) \nRightarrow(\mathrm{CW})$ and hence in this case $(\mathrm{CW})$ is stronger than $(\mathrm{S})$. 


\section{Criteria FOR WEAK CONVERGENCE}

On the $\sigma$-algebra of measurable subsets $e \subset[0,1]$ define for all $\nu \in \mathbb{N} \cup\{0\}$ the functions $\mu_{g^{\nu}}(e):=$ meas $\left(g^{\nu}\right)^{-1}(e)$ and their Radon-Nikodym derivatives

$$
\frac{d \mu_{g^{\nu}}}{d m}(t):=\lim _{\varepsilon \rightarrow 0^{+}} \frac{\mu_{g^{\nu}}([t-\varepsilon, t+\varepsilon])}{2 \varepsilon}, \text { for a.e. } t \in(0,1) .
$$

The methods of the explicit calculation of the latter in various particular cases are given in [1, pp. 21-23]. Also for any $\tau \in[0,1]$ consider the sets

$$
\mathcal{E}_{\nu}(\tau):=\left(g^{\nu}\right)^{-1}([0, \tau])
$$

Further on we will write $\mathcal{E}(\tau)$ instead of $\mathcal{E}_{0}(\tau)$ and omit the reference to $\tau$ when $\tau=1$.

Let $1<q \leq p<+\infty$ and introduce the following set of conditions (the convergence is understood at $\nu \rightarrow \infty)$ :

(Q0) $\left\|\frac{d \mu_{g} \nu}{d m}\right\|_{p /(p-q)} \leq A$ for some $A>0$ and for all $\nu \in \mathbb{N} \cup\{0\}$;

(Q1) $g^{\nu} \rightarrow g$ in measure on $\mathcal{E}$, while meas $\mathcal{E}_{\nu} \Delta \mathcal{E} \rightarrow 0$;

(Q2) meas $\mathcal{E}_{\nu}(\tau) \Delta \mathcal{E}(\tau) \rightarrow 0$ for any $\tau \in[0,1]$.

Here $\Delta$ stands for symmetric difference between the sets. The following theorem contains the main result of this paper.

Theorem 1. The following statements are equivalent:

(i) The operators $S_{g^{\nu}}: L^{p} \rightarrow L^{q}$ converge to $S_{g}: L^{p} \rightarrow L^{q}$ strongly;

(ii) The operators $S_{g^{\nu}}: L^{p} \rightarrow L^{q}$ converge to $S_{g}$ : $L^{p} \rightarrow L^{q}$ weakly;

(iii) Conditions (Q0) and (Q1) hold;

(iv) Conditions (Q0) and (Q2) hold.

Proof. (i) $\Leftrightarrow$ (iii) has been proved in [4]. Furthermore, (i) $\Rightarrow$ (ii) is evident. We will show therefore (ii) $\Rightarrow$ (iv) $\Rightarrow$ (i)

Step 1. (ii) $\Rightarrow$ (iv). If (ii) holds, then the sequence $\left\{S_{g^{\nu}}\right\}, \nu \in \mathbb{N} \cup\{0\}$, is uniformly bounded, which implies (Q0) according to Theorem 1 of [5]. To prove (Q2), choose $x=\chi_{[0, \tau]}$, where $\chi_{e}$ stands for the characteristic function of the set $e \subset[0,1]$. The statement (ii) implies then $\chi_{\mathcal{E}_{\nu}(\tau)} \rightarrow \chi_{\mathcal{E}(\tau)}$ in $L^{q}$, wherefrom (Q2) follows.

Step 2. (iv) $\Rightarrow$ (i). Suppose that (Q0) and (Q2) hold. From (Q0) follows the uniform boundedness of the sequence $\left\{S_{g^{\nu}}\right\}, \nu \in \mathbb{N} \cup\{0\}$. Now observe that the linear span of the set

$$
X_{0}=\left\{\chi_{[0, \tau]} \mid \tau \in[0,1]\right\}
$$

is dense in $L^{p}$. Thus it is enough to show that $S_{g^{\nu}} \chi_{[0, \tau]} \rightarrow S_{g} \chi_{[0, \tau]}$ for all $\tau \in[0,1]$. In fact, taking into account the relationship $S_{g^{\nu}} \chi_{[0, \tau]}=\chi_{\mathcal{E}_{\nu}(\tau)}$ and the fact that for any $f \in L^{q^{\prime}}, 1 / q+1 / q^{\prime}=1$ holds

$$
\begin{aligned}
& \left|\int_{0}^{1} \chi_{\mathcal{E}_{\nu}(\tau)}(t) f(t) d t-\int_{0}^{1} \chi_{\mathcal{E}(\tau)}(t) f(t) d t\right| \leq \\
& \quad\left|\int_{\mathcal{E}_{\nu}(\tau) \backslash \mathcal{E}(\tau)} f(t) d t\right|+\left|\int_{\mathcal{E}(\tau) \backslash \mathcal{E}_{\nu}(\tau)} f(t) d t\right| \rightarrow 0,
\end{aligned}
$$

one proves $S_{g^{\nu}} \chi_{[0, \tau]} \rightarrow S_{g} \chi_{[0, \tau]}$. Moreover, one easily concludes

$$
\left\|S_{g^{\nu}} \chi_{[0, \tau]}\right\|_{q} \rightarrow\left\|S_{g} \chi_{[0, \tau]}\right\|_{q}
$$

thus showing the statement. 


\section{Continuous Weak COnvergence}

Now turn to the continuous weak convergence of a sequence of inner superposition operators. In fact, the previous theorem implies $(\mathrm{CW}) \Rightarrow(\mathrm{W}) \Leftrightarrow(\mathrm{S})$. The following example however shows that $(\mathrm{S}) \nRightarrow(\mathrm{CW})$.

Example I. Let $\alpha>\beta>0, \alpha+\beta=2$ and construct the sequence $g^{\nu}:[0,1] \rightarrow[0,1]$ by the formulae $g^{\nu}(0)=0$ and

$$
\frac{d g^{\nu}}{d t}(t)=\left\{\begin{array}{ll}
\alpha, & k / \nu \leq t<(2 k+1) / 2 \nu, \\
\beta, & (2 k+1) / 2 \nu \leq t<(k+1) / \nu,
\end{array} \quad k=0, \ldots, \nu-1 .\right.
$$

Clearly $g^{\nu} \rightarrow g$, where $g(t)=t$, pointwise, and hence $S_{g^{\nu}}: L^{2} \rightarrow L^{2}$, converge to $S_{g} \equiv I d: L^{2} \rightarrow L^{2}$ strongly ( $I d$ standing for the identity operator). Nevertheless, they do not converge continuously weakly. In fact, supposing the opposite, we would obtain $S_{g^{\nu}} S_{g^{\nu}}^{\prime} \rightarrow I d$ weakly (pointwise) and, according to the previous result, even strongly, while actually

$$
S_{g^{\nu}} S_{g^{\nu}}^{\prime} x-\frac{1}{\alpha \beta} x
$$

We derive now a criterion of a continuous weak convergence of a sequence of inner superposition operators. Introduce for each $\tau \in[0,1], \nu \in \mathbb{N} \cup\{0\}$ the set functions $\mu_{g^{\nu}}(\tau, e):=$ meas $\left(g^{\nu}\right)^{-1}(e) \cap[0, \tau]$ and their Radon-Nikodym derivatives

$$
\frac{d \mu_{g^{\nu}}(\tau)}{d m}(t):=\lim _{\varepsilon \rightarrow 0^{+}} \frac{\mu_{g^{\nu}}(\tau,[t-\varepsilon, t+\varepsilon])}{2 \varepsilon}, \text { for a.e. } t \in(0,1) .
$$

Further on we as usual omit the superscript if $\nu=0$. To calculate the above functions one can use the relationship

$$
\frac{d \mu_{g^{\nu}}(\tau)}{d m}(t)=\frac{d}{d t} \operatorname{meas}\left(g^{\nu}\right)^{-1}([0, t]) \cap[0, \tau] .
$$

It is also worth noting that $\frac{d \mu_{g} \nu(1)}{d m} \equiv \frac{d \mu_{g} \nu}{d m}$. Consider the condition (Q3) $\left\|\frac{d \mu_{g^{\nu}(\tau)}}{d m}\right\|_{p^{\prime}} \rightarrow\left\|\frac{d \mu_{g}(\tau)}{d m}\right\|_{p^{\prime}}$ for each $\tau \in[0,1]$, where $1 / p+1 / p^{\prime}=1$.

Theorem 2. Let $1<q \leq p<+\infty$. Then the following conditions are equivalent:

(i) The operators $S_{g^{\nu}}: L^{p} \rightarrow L^{q}$ converge to $S_{g}: L^{p} \rightarrow L^{q}$ continuously weakly;

(ii) Conditions (Q0), (Q1) and (Q3) hold;

(iii) Conditions (Q0), (Q2) and (Q3) hold.

Proof. The necessity of (Q0) and (Q1) (or (Q0) and (Q2)) for the continuous weak convergence of $S_{g^{\nu}}$ to $S_{g}$ follows from the fact that $(\mathrm{CW}) \Rightarrow(\mathrm{W}) \Leftrightarrow(\mathrm{S})$ for such operators. To show the necessity of $(\mathrm{Q} 3)$ recall that $(\mathrm{CW}) \Leftrightarrow\left(\mathrm{S}^{\prime}\right)$ and note $S_{g^{\nu}}^{\prime} \chi_{[0, \tau]}=\frac{d \mu_{g^{\nu}}(\tau)}{d m}$.

To show the sufficiency of (Q0), (Q1) and (Q3), note that it is enough to prove

(I) The sequence $S_{g^{\nu}}$ has uniformly bounded norms;

(II) $S_{g^{\nu}}^{\prime} y^{\prime} \rightarrow S_{g}^{\prime} y^{\prime}$ for each $y^{\prime} \in X_{0}$, where $X_{0} \subset L^{q^{\prime}}, 1 / q+1 / q^{\prime}=1$, is defined in the proof of Theorem 1 .

Due to Theorem 1 of [5] (I) $\Leftrightarrow(\mathrm{Q} 0)$. Furthermore, from (Q0) and (Q1) it follows that $S_{g^{\nu}}^{\prime} \chi_{[0, \tau]} \rightarrow S_{g}^{\prime} \chi_{[0, \tau]}$ in $L^{p^{\prime}}$, which together with (Q3) implies (II). The sufficiency of (Q0), (Q1) and (Q3) can be shown in a similar way referring to Theorem 1 . 
In practice one often has to deal with the particular case of the operators $S_{g^{\nu}}$ defined on a Hilbert space $L^{2}$. For such a situation we give another useful criterion.

Theorem 3. Let $1<q \leq 2$. Then the following conditions are equivalent:

(i) The operators $S_{g^{\nu}}: L^{2} \rightarrow L^{q}$ converge to $S_{g}: L^{2} \rightarrow L^{q}$ continuously weakly;

(ii) The operators $S_{g^{\nu}}: L^{2} \rightarrow L^{q}$ converge to $S_{g}: L^{2} \rightarrow L^{q}$, while $S_{g^{\nu}} S_{g^{\nu}}^{\prime}: L^{q^{\prime}} \rightarrow$ $L^{q}, 1 / q+1 / q^{\prime}=1$, converge to $S_{g} S_{g}^{\prime}: L^{q^{\prime}} \rightarrow L^{q}$ weakly (pointwise);

(iii) The operators $S_{g^{\nu}}: L^{2} \rightarrow L^{q}$ converge to $S_{g}: L^{2} \rightarrow L^{q}$, while $S_{g^{\nu}} S_{g^{\nu}}^{\prime}: L^{q^{\prime}} \rightarrow$ $L^{q}$ converge to $S_{g} S_{g}^{\prime}: L^{q^{\prime}} \rightarrow L^{q}$ strongly.

Proof. (i) $\Leftrightarrow$ (ii) is the general fact which does not depend on the particular form of the operators. In fact, assuming $(\mathrm{CW})$, one has $\left(\mathrm{W}^{\prime}\right)$, which obviously implies (ii). Conversely, assuming (ii), one has that for any $x^{\prime} \in L^{q^{\prime}}$

$$
\left\langle S_{g^{\nu}} S_{g^{\nu}}^{\prime} x^{\prime}, x^{\prime}\right\rangle=\left\|S_{g^{\nu}}^{\prime} x^{\prime}\right\|_{q} \rightarrow\left\langle S_{g} S_{g}^{\prime} x^{\prime}, x^{\prime}\right\rangle=\left\|S_{g}^{\prime} x^{\prime}\right\|_{q},
$$

which together with (W) implies (i).

The implication (iii) $\Rightarrow$ (ii) is evident. It remains thus to show (i) $\Rightarrow$ (iii). Assuming $(\mathrm{CW})$ one obtains $\left(\mathrm{S}^{\prime}\right)$ and, according to Theorem $1,(\mathrm{~S})$, wherefrom (iii) follows.

The above theorem leads to the following important conclusion on the continuous weak convergence of inner superposition operators generated by invertible functions. We need first an extra definition.

Definition 1. The function $g:[0,1] \rightarrow \mathbb{R}$ is said to satisfy the $\omega$-condition, if there is a measurable function $\gamma:[0,1] \rightarrow \mathbb{R}$ such that $\gamma(g(t))=t$ for a.e. $t \in \mathcal{E}$ and from $e \subset[0,1]$, meas $e=0$ follows meas $\gamma^{-1}(e)=0$.

Corollary 3.1. Let the functions $g^{\nu}, g:[0,1] \rightarrow[0,1]$ satisfy the $\omega$-condition. Then the operators $S_{g^{\nu}}: L^{2} \rightarrow L^{q}, 1<q \leq 2$, converge to $S_{g}: L^{2} \rightarrow L^{q}$ continuously weakly, if and only if the following conditions hold:

(i) $\left\|\frac{d \mu_{g^{\nu}}}{d m}\right\|_{2 /(2-q)} \leq A$ for some $A>0$ and for all $\nu \in \mathbb{N} \cup\{0\}$;

(ii) $g^{\nu} \rightarrow g$ in measure;

(iii) $\frac{d \mu_{g^{\nu}}}{d m}\left(g^{\nu}(\cdot)\right) \rightarrow \frac{d \mu_{g}}{d m}(g(\cdot))$ in $L^{q /(2-q)}\left(*-w e a k l y\right.$ in $L^{\infty}$, if $\left.q=2\right)$.

Moreover, under the above conditions necessarily

$$
\frac{d \mu_{g^{\nu}}}{d m}\left(g^{\nu}(\cdot)\right) \rightarrow \frac{d \mu_{g}}{d m}(g(\cdot)) \text { in } L^{q /(2-q)} .
$$

The proof follows immediately from the representation

$$
\left(S_{g^{\nu}} S_{g^{\nu}}^{\prime} x^{\prime}\right)(t)=\frac{d \mu_{g^{\nu}}}{d m}\left(g^{\nu}(t)\right) x^{\prime}(t) .
$$

\section{REPRESENTATion OF Limit OPERATORS}

We point out that the strong and weak (pointwise) convergence of inner superposition operators are equivalent only under the assumption that the limit operator is also of the same type. In fact, a sequence of inner superposition operators can converge weakly to an operator which cannot be represented in the form (2). 
Example II. Let the functions $g^{\nu}:[0,1] \rightarrow[0,1], \nu \in \mathbb{N}$, be defined by the relationship

$$
g^{\nu}(t):=\left\{\begin{array}{cl}
\nu t, & 1 \leq t<1 / \nu, \\
& \cdots \\
\nu t-k, & k / \nu \leq t<(k+1) / \nu, \quad k=1, \ldots, \nu-1 . \\
& \cdots \\
\nu t-\nu+1, & (\nu-1) / \nu \leq t \leq 1,
\end{array}\right.
$$

Then the respective inner superposition operators $S_{g^{\nu}}: L^{p} \rightarrow L^{p}, 1<p<+\infty$, converge weakly to a limit operator $S: L^{p} \rightarrow L^{p}$ given by the formula

$$
S: x \mapsto \int_{0}^{1} x(t) d t .
$$

To show this it is enough to observe that the operators $S_{g^{\nu}}$ are uniformly bounded, for $\frac{d \mu_{g^{\nu}}}{d m}=1$, and the operator $S$ is continuous, while

$$
S_{g^{\nu}} \chi_{[0, \tau]} \rightarrow S \chi_{[0, \tau]} \text { for any } \tau \in[0,1] .
$$

Moreover, the operators $S_{g^{\nu}}$ converge to $S$ also continuously weakly, because $S_{g^{\nu}}^{\prime}$ converge to $S^{\prime}$ strongly, which can be concluded from the fact that

$$
\left\|S_{g^{\nu}}^{\prime} \chi_{[0, \tau]}\right\|_{p^{\prime}}=\left\|\frac{d \mu_{g^{\nu}}(\tau)}{d m}\right\|_{p^{\prime}} \rightarrow \tau=\left\|S^{\prime} \chi_{[0, \tau]}\right\|_{p^{\prime}}, \quad 1 / p+1 / p^{\prime}=1 .
$$

However, in this example it is clear that the operators $S_{g^{\nu}}$ do not converge strongly. It is also worth noting that the limit operator is compact, while the inner superposition operator is never compact unless it is identically zero [1].

In spite of this discouraging example for the weak convergence of inner superposition operators we have the following representation theorem for a limit of a strongly converging sequence of such operators.

Theorem 4. Let a sequence of inner superposition operators $S_{g^{\nu}}: L^{p} \rightarrow L^{q}$, where $1<q \leq p<+\infty$, generated by the functions $g^{\nu}:[0,1] \rightarrow \mathbb{R}$ converge strongly to an operator $S: L^{p} \rightarrow L^{q}$. Then $S$ is an inner superposition operator generated by some measurable function $g:[0,1] \rightarrow \mathbb{R}$.

Proof. Setting $x(t)=t$ we see that the sequence $\chi_{\mathcal{E}_{\nu}} g^{\nu} \in L^{q}$ converges to some function. In an analogous way, considering $x(t)=1$, we see that also $\chi_{\mathcal{E}_{\nu}} \in L^{q}$ converges to some function, which is necessarily a characteristic function of some measurable set $\mathcal{E} \subset[0,1]$. Now pose by definition $\bar{g}:=\lim _{\nu} \chi_{\mathcal{E}_{\nu}}(t) g^{\nu}$ and

$$
g(t):=\left\{\begin{array}{cl}
\bar{g}(t), & t \in \mathcal{E} \\
2, & t \in[0,1] \backslash \mathcal{E} .
\end{array}\right.
$$

The values of $g^{\nu}(t)$ when $t \notin \mathcal{E}_{\nu}$ do not influence the construction of the operator $S_{g^{\nu}}$, and hence we may assume that at these points $g^{\nu}(t)=2$. Observe that due to the above constructions $\mathcal{E}=g^{-1}([0,1])$ and meas $\mathcal{E}_{\nu} \Delta \mathcal{E} \rightarrow 0$. Thus it is easy to see that $g^{\nu} \rightarrow g$ in measure on $\mathcal{E}$, because

$$
\int_{\mathcal{E}}\left(g^{\nu}(t)-g(t)\right) d t=\int_{\mathcal{E} \backslash \mathcal{E}_{\nu}}\left(g^{\nu}(t)-g(t)\right) d t+\int_{\mathcal{E}}\left(\chi_{\mathcal{E}_{\nu}}(t) g^{\nu}(t)-g(t)\right) d t .
$$

Furthermore, one easily notes that the uniform boundedness of the sequence $S_{g^{\nu}}$ provides the estimate $\left\|\frac{d \mu_{g \nu}}{d m}\right\|_{p /(p-q)} \leq A$. However, at the moment we cannot use 
either Theorem 1 or the results from [4] to show the statement, for it still remains to verify the condition (3) for the limit function.

Suppose the latter are not valid; namely, there exists $e_{0} \subset[0,1]$, meas $e_{0}=0$, such that meas $g^{-1}\left(e_{0}\right)=\beta>0$. Then for any $\varepsilon>0$ there is at most a countable system of nonintersecting open intervals $I, e_{0} \subset I$, such that meas $I<\varepsilon$. Hence

$$
\text { meas } g^{-1}(I) \geq \beta>0 \text {. }
$$

Consider the sequence of sets $A_{\nu}:=\left(g^{\nu}\right)^{-1}(I) \cap \mathcal{E}$. One observes that it contains a subsequence $A_{\nu_{\mu}}$ which tends to $g^{-1}(I)$ in the following sense [6]:

$$
\bigcap_{n=1}^{\infty}\left(\bigcup_{\mu=n}^{\infty} A_{\nu_{\mu}}\right)=\bigcup_{n=1}^{\infty}\left(\bigcap_{\mu=n}^{\infty} A_{\nu_{\mu}}\right)=g^{-1}(I)
$$

(the equality is understood up to some subset of zero measure). Thus

$$
\text { meas } g^{-1}(I)=\lim _{\mu} \text { meas } A_{\nu_{\mu}} \text {, }
$$

but the uniform estimate $\left\|\frac{d \mu_{g} \nu}{d m}\right\|_{p /(p-q)} \leq A$ implies meas $A_{\nu} \rightarrow 0$ when $\varepsilon \rightarrow 0$ uniformly with respect to $\nu$, which leads to a contradiction.

Having demonstrated the fulfillment of the condition (3) we in fact have shown the statement. Namely, it remains to refer to Lemma 2 from [4], which asserts that $S_{g^{\nu}} x \rightarrow S_{g} x$ in measure, observing that then necessarily $S_{g} x=S x$.

\section{ACKNOWLEDGEMENTS}

We are grateful to Dr. L. Freddi (Università di Udine, Italy) and to Prof. G. Buttazzo (Università di Pisa, Italy) for interesting suggestions.

\section{REFERENCES}

1. N. V. Azbelev, V. P. Maksimov, and L.F. Rakhmatullina. Introduction to the Theory of Functional Differential Equations. "Nauka", Moscow, 1991. in Russian. MR 92j:34123

2. G. Buttazzo and L. Freddi. Optimal control problems with weakly converging input operators. Discrete and Continuous Dynamical Systems, 1(3):401-420, 1995. MR 97b:49016

3. C. Kuratowski. Topologie, volume 1. Państwowe Wydawnictwo Naukowe, Warszawa, 1958. in French. MR 19:873d

4. M.E. Drakhlin. On convergence of sequences of internal superposition operators. Functional Differential Equations. Israel Seminar, 1:83-94, 1993. MR 95g:47047

5. M.E. Drakhlin. An inner superposition operator in the spaces of summable functions. Izvestiya VUZ. Matematika, 30(5):18-24, 1986. in Russian. MR 88b:47039

6. Lusternik L. A. and Sobolev V. I. Short Course of Functional Analysis. Vyschaya Shkola, Moscow, 1982. in Russian.

Research Institute, College of Judea and Samaria, Kedumim-Ariel, D.N. Efraim 44820, ISRAEL

Scuola Normale Superiore, Piazza dei Cavalieri 7, 56126 Pisa, Italy

E-mail address: stepanov@cibs.sns.it 